\title{
Sexual Differences in Gonad Fatty Acid Compositions in Dominant Limpets Species from the Sanriku Coast in Northern Japan
}

\author{
Hideki Kawashima $^{1 *}$, Masao OHNishi ${ }^{2}$ and Hiroo UChIYAMa ${ }^{3}$ \\ ${ }^{1}$ Bioscience Laboratory, Miyako College Division, Iwate Prefuctural University \\ (1-5-1, Ka-nan, Miyako-shi, Iwate 027-0039, JAPAN) \\ ${ }^{2}$ Department of Bioresource Science, Obihiro University of Agriculture and Veterinary Medicine \\ (Inada-cho, Obihiro-shi, Hokkaido 080-8555, JAPAN) \\ ${ }^{3}$ Water and Soil Environment Division, National Institute for Enviromental Studies \\ (16-2, Onogawa, Tsukuba-shi, Ibaraki 305-0053, JAPAN)
}

Edited by H. Saito, Nat. Res. Fish Sci., and accepted February 25, 2002 (received for review December 27, 2001)

\begin{abstract}
Lipids in the gonads of two species of limpets from the Sanriku Coast in northern Japan were examined for lipid class and fatty acid composition. Dominant limpets were found to contain total lipids (TL) at, 1 to $2 \%$ and 5 to $6 \%$, in the testes and ovaries, respectively. The testis was rich in phosphatidylcholine and phosphatidylethanolamine was found present. Triacylglycerol (TG) was the main constituent in the ovary. Significant differences were found in the proportion of fatty acid components of TL for those two organs. Icosapentaenoic acid (20:5n3 ) was the most abundant fatty acid in the testis, with palmitic acid (16:0) being so in the ovary. Predominant fatty acids ( $>10 \%$ of total fatty acids in TL) generally contained 16:0, vaccenic acid $(18: 1 n-7)$ and $20: 5 n-3$ and 16:0, 18:1n-7 and arachidonic acid (20:4n-6) in the testis and ovary, respectively. The testis tends to be predominantly with amount of n-3 polyunsaturated fatty acids, 20:5n-3 being particularly high, at twice that in the ovary. The ovary contained much higher saturated fatty acids, compared to testis lipids. The quantitative distribution of fatty acids in lipid classes for male and female gonads was also determined. TG in the testis and ovary were rich in saturated fatty acid with abundant 16:0. The proportions of 20:4n-6 and 20:5n-3 in TG were low compared with those in TL and phospholipids (PL). PL contained more 20:4n-6 and 20:5n-3 than TG. PL in the testis were rich in 16:0, 18:1n-7 and 20:5n-3, and rich in 16:0, 20:4n-6 and 20:5n-3 in the ovary.
\end{abstract}

Key words: limpet, Cellana grata, Collisella dorsuosa, gonad lipid, polyunsaturated fatty acid

\section{Introduction}

Limpets are gastropods frequently found on the tidal rocks on many coastline throughout the world. In Japan, various species of limpets are abundantly present through the year. To date, though ecological and taxonomic studies have been primarily conducted on limpets, biochemical and physiological data on the reproductive cycle of this gastropod are few. Some determination has been made of fatty acid compositions

\footnotetext{
*Correspondence to: Hideki Kawashima, Bioscience Laboratory, Miyako College Division, Iwate Prefuctural University, 1-5-1, Ka-nan, Miyako-shi, Iwate 027-0039, JAPAN

E-mail: ajoe@iwate-pu.ac.jp
} 
of whole gonads without regard to limpet sex (1-3). Still, this parameter in the testis and ovary in limpets remains essentially unclarified, except for the Turbinidae family; Subninella undulata (1). The present study was undertaken to determine and compare lipid classes and fatty acid compositions for these two organs in dominant species of limpets during maturation on the Sanriku Coast. Special attention was directed to the distribution of fatty acid components in major lipids classes for gonads of both sexes. This distribution in lipid classes was also examined for comparison. The authors consider that the results obtained should significantly contributed to an understanding of the reproductive cycle in marine invertebrates.

\section{Materials and Methods}

Dominant species of Japanese limpets, Cellana grata (C.grata) and Collisella dorsuosa (C. dorsuosa) obtained in August, 2001 from Miyako and Funakoshi Bays, Iwate Prefecture, in northern Japan, and transported to the laboratory where the gonads were immediately removed and placed on ice. Sex was determined by microscopic examination, and the gonads classified as either testes (male gonads) or ovaries (female gonads). Gonads were homogenized and lipids extracted in accordance with the method of Bligh and Dyer (4). Total lipid determination was made based on thinlayer chromatography (TLC) (5) using Silica gel 60 plates (Merck, Darmstadt, Germany) with a mixture of $n$-hexane-diethyl ether-acetic acid (80:20:1, v/v/v) and chloroform-methanol-water $(65: 25: 4, \mathrm{v} / \mathrm{v} / \mathrm{v})$ as the mobile phase. Lipids were detected by charring with $50 \%$ sulfuric acid or Dittmer reagent (6).

Fractionation of TL into neutral lipids, polar lipids, triacylglycerol (TG) and phospholipids (PL) was conducted by preparative TLC on Pre-Coated TIC plates (Silica gel 60) with the same developing solvent systems specified above. Each fractionated spot detected by $\mathrm{I}_{2}$ vapor was scraped from the TLC plate and extracted twice with a chloroform-methanol $(2: 1, \mathrm{v} / \mathrm{v})$ solution. The percentages of neutral and polar lipids were expressed as amounts of total fatty acids in each lipid.

Fatty acid methyl esters were prepared using boron trifluoride-methanol (7) and analyzed by capillary gasliquid chromatography (GC) subsequent to the purification by TLC with a $n$-hexane-diethyl ether $(8: 2, \mathrm{v} / \mathrm{v})$ solution (8). GC analysis was conducted using a Shimadzu GC-8A gas chromatograph provided with a flame ionization detector and a fused silica open tubular column, SUPELCOWAX-10 (0.25 mm i.d. $\times 30 \mathrm{~m}$, $0.25 \mu \mathrm{m}$ film thickness, Sigma Aldrich Japan), operated isothermally at $210^{\circ} \mathrm{C}$. The carrier gas was helium at a flow rate of $1.0 \mathrm{~mL} / \mathrm{min}$. Fatty acid peaks were identified based on comparison of retention times with authentic standards (Sigma Aldrich Japan and Supelco), with confirmation made by GC-MS analysis. GC-MS was carried out on fatty acid methyl esters with a Hewlett-Packard HP 5972 SERIES, on a fused silica open tubular capillary column (same as for GC) under conditions as the same for GC. Ionization energy was $70 \mathrm{eV}$. Analysis in all cases was conducted in duplicate

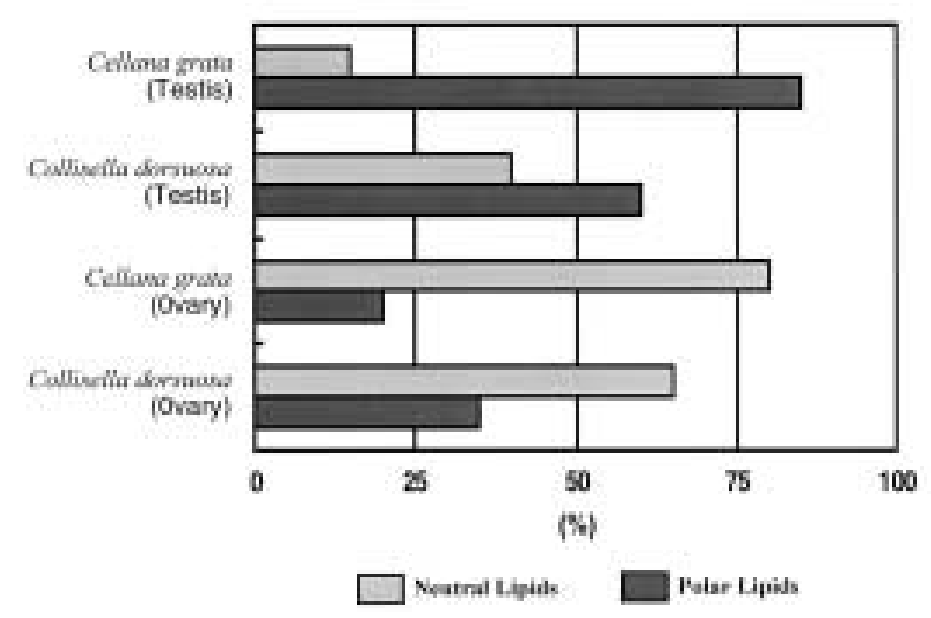

Fig. 1 Neutral and Polar Lipid Contents in Gonads for Two Limpets Species from Sanriku Coast. 
and the results expressed as mean values.

\section{Results and Discussion}

Lipid content in testes and ovaries from mature gonads in Japanese limpets collected in August, 2001 ranged from 1 to $2 \%$, and 5 to $6 \%$ of wet tissue weight, respectively (data not shown). The proportions of neutral and polar lipids in the testis and ovary are shown in Fig. 1. Percentages of polar lipids of the testis and neutral lipids of the ovary were more than 60 and $65 \%$, respectively. Major components of TL were triacylglycerol (TG), phosphatidylcholine (PC) and phosphatidylethanolamine (PE), with being sterols and sterol esters present in minor amounts. TG (58-63\%) was the main lipid component in the ovary, and PC (33$37 \%)$ and PE (30-32\%) predominant lipid class in the testis (data not shown).

Fatty acid compositions of TL from the testis and ovary of the dominant species are listed in Table 1. Several fatty acid peaks, including unidentified ones, were also detected, but not regarded in this study because of their trace amounts. Each fatty acid displayed a unique profile. Icosapentaenoic acid (20:5n-3) was most abundant fatty acid in the testis, and palmitic acid (16:0) in the ovary. n-6 and n-3 polyunsaturated fatty acids (PUFA) were the most abundant unsaturated fatty acids, with arachidonic acid (20:4n-6) and 20:5n-3

Table 1 Fatty Acid Compositions of Testis and Ovary Lipids in Two Limpets Species from Sanriku Coast in Northern Japan. (Aug., 2001)

\begin{tabular}{|c|c|c|c|c|}
\hline \multirow{2}{*}{ Fatty Acid } & \multicolumn{2}{|c|}{ Testis } & \multicolumn{2}{|c|}{ Ovary } \\
\hline & Cellana grata & Collisella dorsuosa & Cellana grata & Collisella dorsuosa \\
\hline $\mathrm{C} 14: 0$ & 1.4 & 5.4 & 9.1 & 6.3 \\
\hline C14:1n-5 & 0.4 & 0.7 & 0.6 & 0.6 \\
\hline $\mathrm{C} 16: 0$ & 19.0 & 17.6 & 29.1 & 22.8 \\
\hline C16:1n-7 & 3.1 & 3.8 & 3.9 & 4.5 \\
\hline $\mathrm{C} 16: 1 \mathrm{n}-5$ & $<0.1$ & $<0.1$ & $<0.1$ & $<0.1$ \\
\hline C18:0 & 5.9 & 7.5 & 4.8 & 4.3 \\
\hline C18:1n-9 & 2.1 & 5.4 & 8.6 & 7.1 \\
\hline C18:1n-7 & 17.8 & 16.4 & 14.5 & 15.8 \\
\hline C18:2n-6 & 0.4 & $<0.1$ & 2.4 & 0.7 \\
\hline C18:3n-6 & 0.4 & 3.7 & $<0.1$ & 2.2 \\
\hline C18:3n-3 & 1.5 & 0.5 & 3.2 & 0.5 \\
\hline C18:4n-3 & $<0.1$ & $<0.1$ & $<0.1$ & $<0.1$ \\
\hline $\mathrm{C} 20: 0$ & $<0.1$ & $<0.1$ & $<0.1$ & 3.2 \\
\hline C20:1n-9 & 2.6 & $<0.1$ & 3.5 & 2.1 \\
\hline C20:1n-7 & $<0.1$ & $<0.1$ & $<0.1$ & 0.8 \\
\hline$C 20: 2 n-6$ & $<0.1$ & 1.8 & $<0.1$ & 2.4 \\
\hline$C 20: 3 n-6$ & 2.0 & 5.1 & $<0.1$ & $<0.1$ \\
\hline$C 20: 4 n-6$ & 2.6 & 6.6 & 12.7 & 14.9 \\
\hline$C 20: 4 n-3$ & $<0.1$ & $<0.1$ & $<0.1$ & 1.5 \\
\hline C20:5n-3 & 40.8 & 25.4 & 7.7 & 10.2 \\
\hline C22:1n-9 & $<0.1$ & $<0.1$ & $<0.1$ & $<0.1$ \\
\hline$C 22: 4 n-6$ & $<0.1$ & $<0.1$ & $<0.1$ & $<0.1$ \\
\hline Sat FA & 26.3 & 30.5 & 43.0 & 36.6 \\
\hline Unsat FA & 73.7 & 69.5 & 57.0 & 63.4 \\
\hline n-6 PUFA* & 5.4 & 17.2 & 15.1 & 20.2 \\
\hline n-3 PUFA & 42.3 & 25.9 & 10.9 & 12.2 \\
\hline UI** & 2.52 & 2.09 & 1.35 & 1.62 \\
\hline
\end{tabular}

*Polyunsaturated fatty acid.

$* *\left(\Sigma \mathrm{m}_{i} \cdot \mathrm{n}_{i}\right) /(100)$, where $\mathrm{m}_{i}$ is percentage and $\mathrm{n}_{i}$, number of C-C double bonds of fatty acid $i$. 
present in the greatest amounts, followed by total saturated and monounsaturated fatty acids (MUFA), of which $16: 0$ and vaccenic acid (18:1n-7) were the most abundant.

Comparison of fatty acid compositions in the testis and ovary indicated unsaturated fatty acid content to be greater in the former, with n-3 PUFA present at twice that in the latter. The testis contained significant amounts of polyenes (C. grata and C. dorsuosa, 48 and $43 \%$, respectively) with $20: 4 n-6$ and $20: 5 n-3$ as major components, compared to the ovary. Fatty acid compositions in the testis and ovary are thus shown to differ considerably, with much greater amounts of 20:5n-3 than 20:4n-6 present in the former. Total n-3 PUFA was richer in male than female gonads. 16:0 was significantly more abundant in the ovary.

The unsaturation index (UI, average number of double bonds per acyl chain) (8) as determined from the present findings is thus markedly higher in the testis than in the ovary. (C. grata and C. dorsuosa, 2.52 and 2.09 in the testis compared to 1.35 and 1.62 in the ovary, respectively). Predominant fatty acids $(10 \%>$ of total fatty acids in TL) were 16:0, 18:1n-7 and 20:5n3 , and $16: 0,18: 1 n-7,20: 4 n-6$ and $20: 5 n-3$, in the testis and ovary, respectively. 20:5n-3 was more in the testis of $C$. grata than in C. dorsuosa. But in either, 20:5n-3 content exceeded that of 20:4n-6. In the ovary $16: 0$ was the most abundant fatty acid (C. grata and C. dorsuosa, 29 and $23 \%$, respectively). Unsaturated fatty acids in the testis are shown by the present results to be for more abundant than in the ovary, with 20:4n-6 and 20:5n-3 content clearly differing in the gonads of either sex. To our knowledge, this paper presents for the first time differences in fatty acid composition in the gonads of the two both sexes of $C$. grata and $C$. dorsuosa belonging to the Nacellidae and Lottidae family, respectively.

Johns et al. determined fatty acid compositions of male and female gonads from the Australian gastropod, Subninella undulata ( $S$. undulata), belonging to the Turbinidae family and found predominant fatty acids $(10 \%>$ of total fatty acids in TL) from the testis of $S$. undulata to be 16:0, 20:4n-6, 20:5n-3 and 22:5n-3, present at $38 \%$ and predominant fatty acid in the ovary to be $16: 0$ and $18: 1 n-9$ at $33 \%$ (1). UI of the main fatty acids of the testis in $S$. undulata is thus shown by the present study higher than in the ovary (UI in the testis and ovary, 1.87 and 1.42, respectively). The high degree of unsaturation was found essentially the same for $C$. grata and C. dorsuosa. 22:5n-3, however, could not be detected and 18:1n-7 content in C. grata and C. dorsuosa was much greater than in S. undulata. 20:5n-3 in total fatty acids was found most abundant in the testis of dominant limpets species from the Sanriku Coast in northern Japan. n-7 MUFA was higher in the gonads. The function of n-7 MUFA in limpet gonads is not understood at present. It is evident from this study that predominant fatty acid composition in gonad lipids of Japanese limpets remarkably differs from that of Australian species (1). But in contrast to gonad lipids from limpets, unsaturated fatty acids such as 20:5n-3 and docosahexaenoic acid (22:6n-3), at $45 \%$ of total fatty acids, of the female mussel gonad from Mytilus californianus were present in much greater amounts than in the male (UI of the testis and ovary, 1.78 and 2.62, respectively) (9). Component fatty acids of gonad lipids are thus shown to differ according to sex for bivalve and limpet species. The relation between fatty acid composition of gonad lipids from limpets and physiological roles of PUFA such as 20:4n-6 and 20:5n-3 in reproductive cycle should be more fully clarified.

To determine fatty acid component distribution in major lipid classes for gonads of both sexes, fatty acid compositions of those classes were analyzed for comparison with gonad lipids. The results of TG and PL (mainly PE and PC) fatty acid component comparison for the testis and ovary from two limpets species are shown in Fig. 2. Major saturated fatty acid and MUFA in both classes were 16:0 and 18:1n-7, respectively. In TG 16:0 was most abundant, followed by $18: 1 \mathrm{n}-7$ and 18:1n-9, indicating high 16:0 content. A amount of the fatty acids in the ovary exceeded those in the testis. 16:0 of TG in C. grata was higher than in C. dorsuosa. Differences in predominant saturated fatty acid of TL in the ovary may thus possibly arise from those in the amounts of TG. PUFA was significantly more in PL than TG and total PUFA of PL, in $C$. dorsuosa and $C$. grata, was 36 and $48 \%$ of total fatty acids, respectively. $20: 5 n-3$, present somewhat more than $20: 4 n-6$, was most abundant in PL of the testis. 20:4n-6 content was highest in PL of the ovary. 18:1n-7 and 20:5n-3 were high in the testis, with 20:4n-6 content being higher in the ovary as shown in Fig. 2. Total amount of 20:4n-6 and 20:5n-3 reached nearly $50 \%$ of total fatty acids in the ovary of $C$. dorsuosa. PL contained more 20:4n-6 than TG. This fatty acid is a major precursor of prostaglandins which influences reproduction in the 

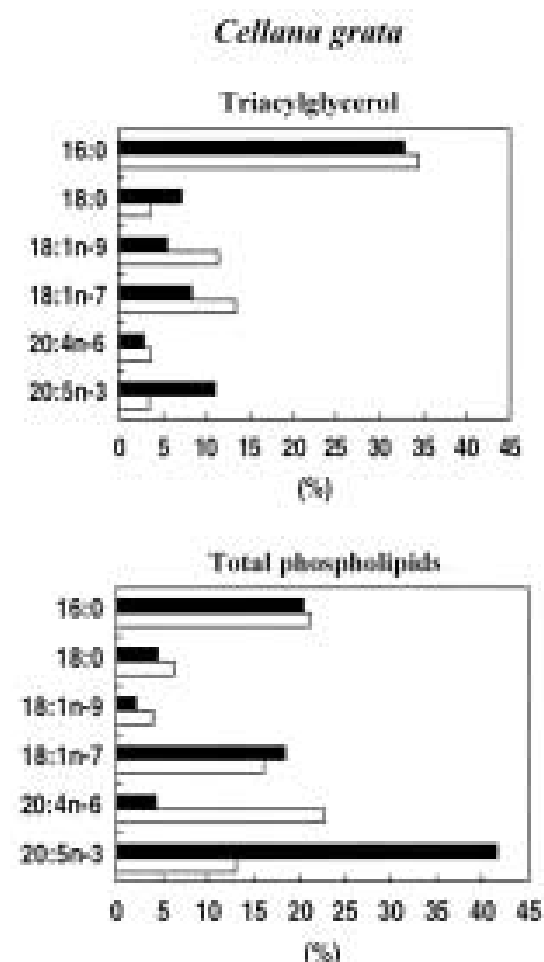

(\%)
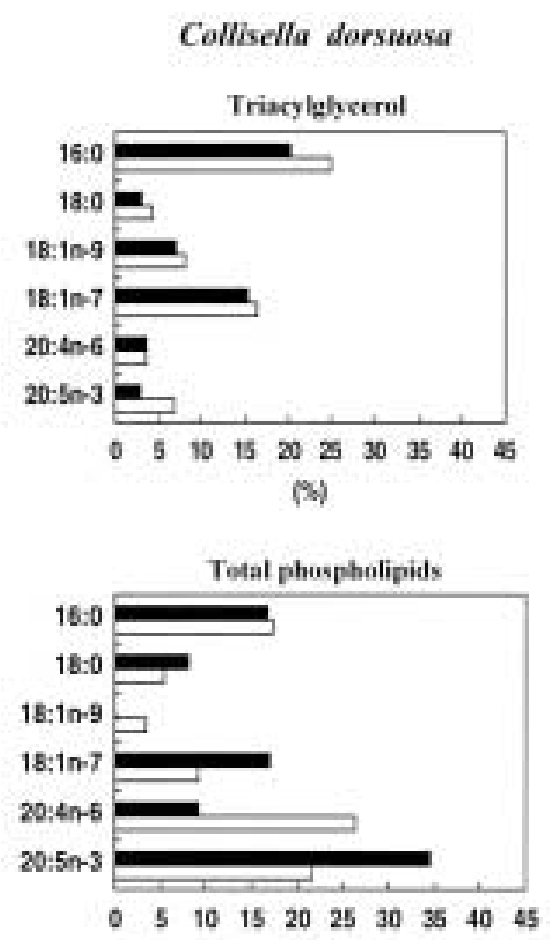

- Testis

口ovary

Fig. 2 Comparison of Fatty Acid Components in Triacylglycerol and Total Phospholipids between Testis and Ovary.

scallop, Patinopecten yessoensis (10). Marine PL is usually more abundant in PUFA than NL (11). UI of PL (based on predominant fatty acids, 1.77-2.46) in both sexes remarkably exceeded that of TG (based on predominant fatty acids, $0.52-0.81$ ), this being due primarily to the high 20:4n-6 and 20:5n-3 content in PL. Differences in the amounts of n-3, n-6 PUFA and MUFA amount of TL in male and female gonads may thus possibly arise from those in PL, as noted in this study.

The present differences in fatty acid compositions in the testis and ovary of limpets according to sex may be to a clue for understanding not only the reproductive cycle in marine invertebrates but also mechanisms of biologically active lipid metabolites known as eicosanoids that are essential to reproductive physiology (12-14).

\section{Acknowledgments}

This work was supported by a Grant-in-Aid for the Research Program, "Studies on chemical ecology of gastropods inhabiting the littoral zone of the Sanriku
Coast, northern Japan" of the Iwate Prefectural Foundation of Academic Research Promotion. The authors thank Professor N. Miyazaki, University of Tokyo, for continuous encouragement throughout this work. The present work was partially conducted as a cooperative research program with the Otsuchi Marine Research Center, Ocean Research Institute, University of Tokyo.

\section{References}

1. Johns, R. B., Nichols, P. D. and Perry. G. J. (1980) Comp. Biochem. Physiol., 65 B, 207-214.

2. Joseph, J. D. (1982) Progress in Lipid Research (Holman, R.T., ed.) Vol. 21, Pergamon Press Ltd., London, pp. 109-153.

3. Joseph, J. D. (1989) Marine Biogenic Lipids, Fats, and Oils (Ackman, G. E., ed.) Vol. II, CRC Press Inc., Florida, pp. 49143.

4. Bligh, E. G. and Dyer, W. J. (1959) Can. J. Biochem. Physiol., 37, 911-917.

5. Skidmore, W. D. and Entenman, C. (1962) J. Lipid Res., 4, 471475.

6. Dittmer, J. C. and Lester, R. L. (1964) J. Lipid Res., 5, 126-127.

7. Morrison, W. R. and Smith, L. M. (1964) J. Lipid Res., 5, 600- 


\section{H. Kawashima, M. Ohnishi and H. Uchiyama}

608.

8. Kawashima, H., Ohnishi, M. and Uchiyama, H. (2001) J. Oleo Sci., 50, 607-611.

9. Rodegker, W. and Nevenzel, J.C. (1964) Comp. Biochem. Physiol., 11, 53-60.

10. Osada, M., Nishikawa, M. and Nomura, T. (1989) Comp. Biochem. Physiol., 94C, 595-601.

11. Vaskovsky, V. E. (1989) Marine Biogenic Lipids, Fats, and Oils
(Ackman, G. E., ed.) Vol. II, CRC Press Inc., Florida, pp. 199242.

12. Coffa, G. and Hill, E. M. (2000) J. Am. Oil Chem. Soc., 35, 1195-1204.

13. Hill, E. M., Holland, D. L. and East, J. (1993) Biochim. Biophys. Acta, 1157, 297-303.

14. Hada, T., Swift, L. L. and Brash, A. R. (1997) Biochim. Biophys. Acta, 1346, 109-119. 